\title{
Photonic microstructures as laser mirrors
}

\author{
Thomas F. Krauss \\ California Institute of Technology \\ Department of Electrical Engineering \\ Mail Code 136-93 \\ Pasadena, California 91125 \\ and \\ University of Glasgow \\ Department of Electronics and Electrical \\ Engineering \\ Glasgow, Scotland G12 8LT \\ United Kingdom \\ E-mail: tfk@cco.caltech.edu

\section{Oskar Painter} \\ Axel Scherer \\ California Institute of Technology \\ Department of Electrical Engineering \\ Mail Code 136-93 \\ Pasadena, California 91125 \\ John S. Roberts \\ University of Sheffield \\ EPSRC III-V Semiconductor Growth Facility \\ Sheffield, England S1 3JD \\ United Kingdom \\ Richard M. De La Rue \\ University of Glasgow \\ Department of Electronics and Electrical \\ Engineering \\ Glasgow, Scotland G12 8LT \\ United Kingdom
}

\begin{abstract}
Deeply etched 1-D third-order Bragg reflectors have been used as mirrors for broad-area semiconductor lasers operating at 975-nm wavelength. From a threshold and efficiency analysis, we determine the mirror reflectivity to be approximately $95 \%$. The design of the GaAs-based laser structure features three InGaAs quantum wells placed close $(0.5 \mu \mathrm{m})$ to the surface in order to reduce the required etch depth and facilitate high-quality etching. Despite the shallow design and the proximity of the guided mode to the metal contact, the threshold current density $\left(J_{\text {th }}=220 \mathrm{~A} / \mathrm{cm}^{2}\right.$ for infinite cavity length) and internal loss $\left(\alpha_{i}=9 \pm 1 \mathrm{~cm}^{-1}\right)$ are very low. (c) 1998 Society of Photo-Optical Instrumentation Engineers. [S0091-3286(98)00404-8]
\end{abstract}

Subject terms: photonic microstructures; semiconductor lasers; Bragg mirrors; photonic bandgap; low threshold.

Paper INT-04 received Aug. 19, 1997; revised manuscript received Oct. 21, 1997; accepted for publication Oct. 27, 1997.

\section{Introduction}

Periodic microstructures that consist of alternating layers of semiconductor material and air can be regarded as the extreme limit of the type of Bragg grating commonly used in distributed feedback (DFB) and distributed Bragg reflector (DBR) lasers. The difference is the refractive index contrast, which is typically less than $1 \%$ in a DFB/DBR laser, but as large as 3.5:1 in the case discussed here. This leads to a very much shorter interaction length of around $1 \mu \mathrm{m}$, instead of the hundreds of microns in a DFB/DBR laser, and thereby opens the opportunity of creating edgeemitting laser elements with very small optical volume. This degree of compactness and the associated capability of very low threshold and high frequency operation has hitherto been reserved for vertical cavity surface emitting lasers (VCSELs), where thresholds below $100 \mu \mathrm{A}$ have already been achieved. ${ }^{1}$

Our semiconductor-air grating can also be regarded as the simplest realization of a photonic bandgap (PBG) structure $^{2}$ (or "photonic crystal"), because it fulfills the requirements of high refractive index contrast and small $(<100 \mathrm{~nm})$ feature size that are essential for PBGs in two and three dimensions. Furthermore, this is one of the few examples $^{3-6}$ where a photonic microstructure has been created and successfully used in an active laser material.
Ultimately, it will be possible to realize light-emitting devices where the active area is totally enclosed in a photonic crystal and will therefore have its emission characteristic radically altered; the high-reflectivity mirror described here is only a first, albeit very important, step towards this goal. We have purposely restricted ourselves to a onedimensional structure, because in principle it only reflects and transmits light; two-dimensional lattices, in addition, exhibit in-plane diffraction ${ }^{7}$ (where the light remains confined to the waveguide plane but changes direction of travel, as opposed to out-of plane diffraction, which is due to fabrication imperfections and the limited depth of the structure, and leads to scattering of light into the substrate and the air space above the waveguide). In-plane diffraction is undesirable for simple laser mirrors, but, when carefully controlled, could be employed for mode control or for interaction between different elements of an integrated circuit. Also, the wide angular bandwidth of a 2-D structure is not required in an edge-emitting laser, particularly if the laser operates in a single transverse mode.

The motivation for this work is thus to (1) demonstrate the use of a very compact, high-reflectivity mirror as the feedback element for a DBR semiconductor laser and (2) confirm the previous transmission measurements ${ }^{8,9}$ in re- 

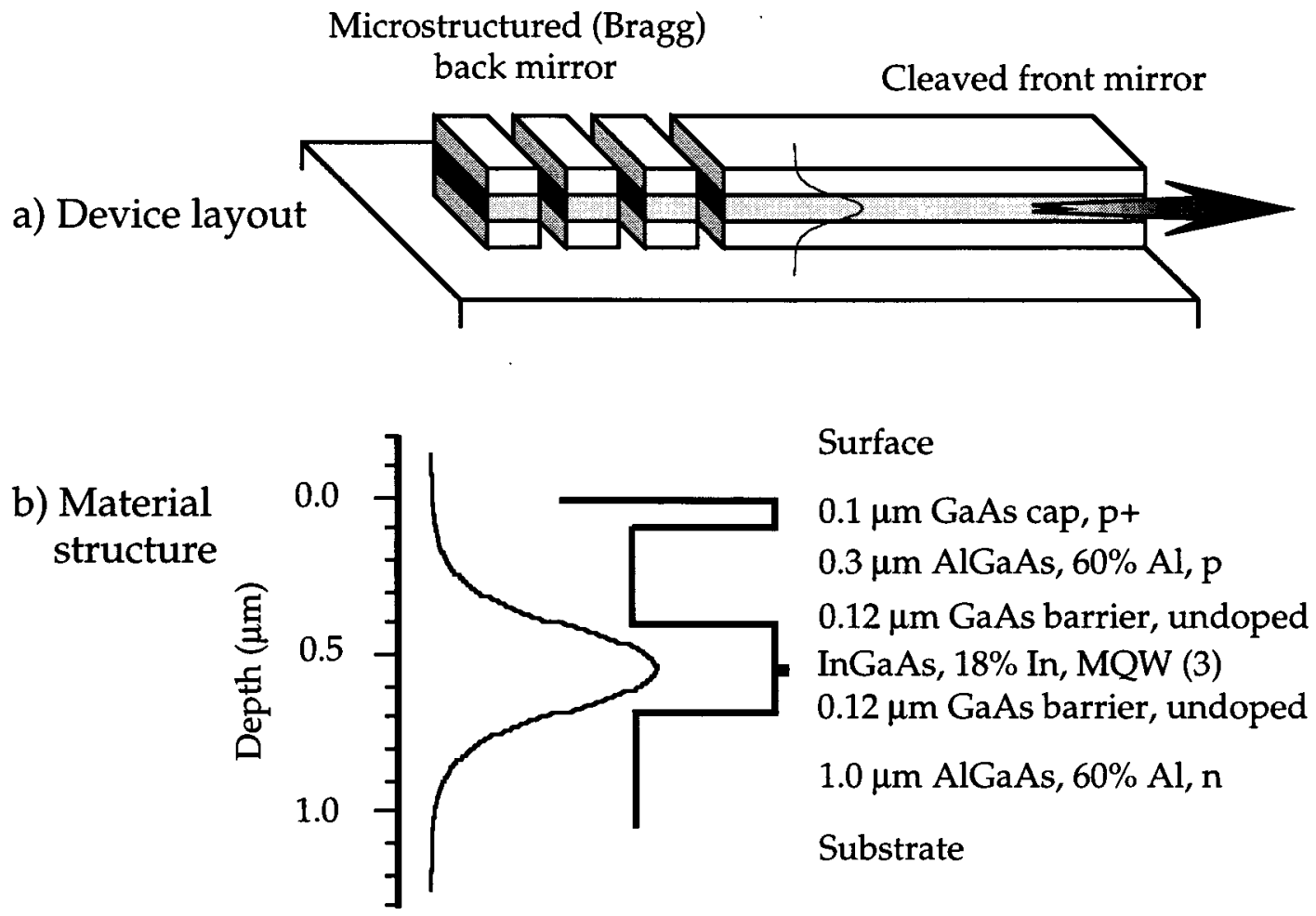

Fig. 1 (a) Sketch of the DBR laser cavity with microstructured back mirror and cleaved front mirror. (b) Design of the epitaxial structure, using a GaAs/AlGaAs waveguide and three InGaAs quantum wells. The waveguide is designed to maximize the confinement of the mode in the core region and to minimize the penetration of the mode tails into the substrate and the top metal contact. The mode shape was modeled with a finite difference routine.

flection. The cavity layout is shown in Fig. 1(a), and the actual device in Fig. 2.

\section{Design}

As discussed previously, ${ }^{10}$ we believe that the best design for a waveguide-based photonic microstructure consists of a lattice with a small percentage of air. Such a lattice requires etching small holes or slots, which puts a high demand on the quality of the dry-etch process that is used to

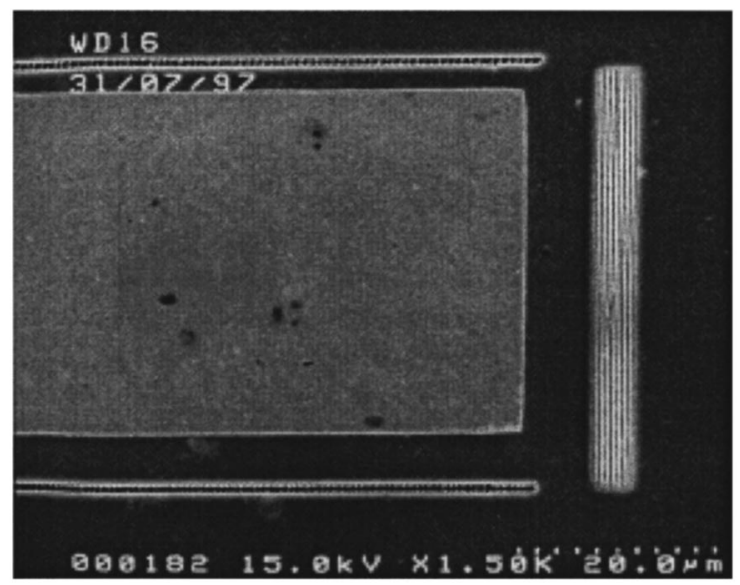

Fig. 2 Top view of a laser with a microstructured back mirror. fabricate these features. Due to the intricate relationship between resolution, mask thickness, and achievable etch depth, we found a depth limit of about $1 \mu \mathrm{m}$ for the narrow slots etched in the present work, where a silicon dioxide mask was used; greater depths are possible with a mask consisting of oxidized AlAs, ${ }^{5}$ but those masks require a more elaborate fabrication process.

Hence, the waveguide must be designed so that the guided mode is entirely contained within the top $1 \mu \mathrm{m}$ of the material and so that the mode decays rapidly towards the top surface. The latter constraint minimizes the interaction with the metal contact that is necessary for current injection. The design shown in Fig. 1(b) fulfills both of these requirements, as shown by the profile of the guided mode and the fact that the internal loss is smaller than $10 \mathrm{~cm}^{-1}$ (see below for details). This design contrasts with the standard layout of semiconductor lasers, where typically, $1.0-$ to $1.5-\mu \mathrm{m}$ claddings are used; such thick claddings were initially designed for much longer lasers, where the internal loss, partly caused by mode interaction with the metal contact, is a more important issue. Our work, in contrast, is aimed at much shorter devices that are dominated by the mirror loss, so the small increase in the internal loss resulting from moving the active layer closer to the surface can be tolerated. Furthermore, we increased the thickness of the guiding layer, which leads to stronger confinement of the mode and a more rapid decay of its tail in the cladding. This principle of a thick-surface waveguide has already 


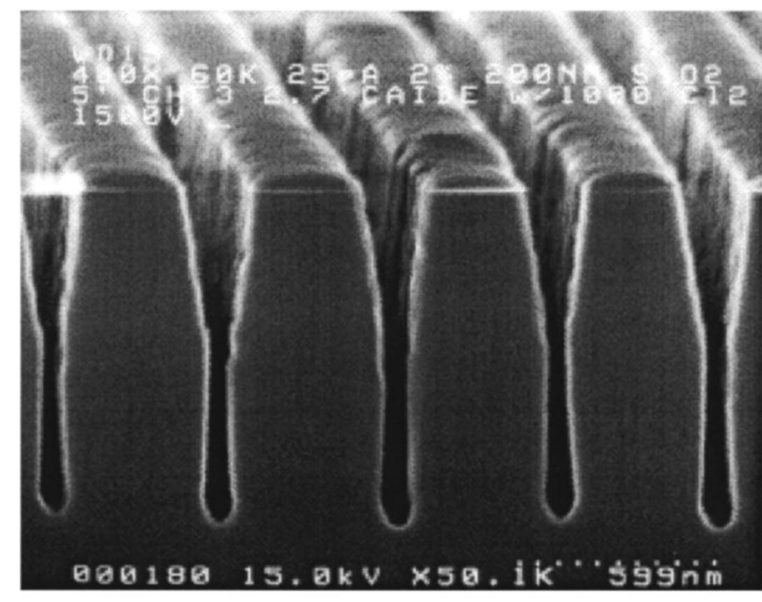

Fig. 3 Cross section of a microstructured Bragg mirror. The etch depth is $1 \mu \mathrm{m}$, the period is $500 \mathrm{~nm}$, and the etched slots are 60 to $100 \mathrm{~nm}$ wide.

been used successfully in previous, passive experiments. ${ }^{8,9}$

A period of $500 \mathrm{~nm}$ was chosen for the microstructure. Considering the average width of the etched slots of approximately $80 \mathrm{~nm}$ (Fig. 3), this combination should result in a third-order stopband extending from approximately 920- to 1080-nm wavelength, which comfortably includes the lasing wavelength of $975 \mathrm{~nm}$.

\section{Fabrication}

The epitaxial laser structure was grown by metal-organic chemical vapor deposition. The laser contacts and microstructured mirrors were defined by electron-beam lithography, using a converted ISI scanning electron microscope (SEM). The top, $p$-type contacts (Ni-Au) were defined first, followed by the sputter deposition of a 200-nm-thick layer of $\mathrm{SiO}_{2}$. The high-resolution pattern was then defined in 70-nm-thick electron-beam resist and transferred into the $\mathrm{SiO}_{2}$ by reactive ion etching (RIE) using $\mathrm{CHF}_{3}$. Transfer of the pattern into the semiconductor was achieved by chemically assisted ion beam etching (CAIBE) using $\mathrm{Cl}_{2}$ as the reactive gas and a beam voltage of $1500 \mathrm{~V}$. Finally, the $n$-type contact (Au-Ge-Ni) was deposited on the back of the substrate. The contacts were not annealed.

\section{Results and Analysis}

The stripes (43 $\mu \mathrm{m}$ wide) were cleaved into lasers of different lengths (between 88 and $450 \mu \mathrm{m}$ long), so the front mirror was formed by a cleaved facet and the back mirror by the microstructure; simple etched mirrors (i.e., ones where a single dry-etched semiconductor-air interface acts as the back mirror) were included for comparison. Devices were operated pulsed (3- $\mu$ s pulses), and the $L-I$ curves were recorded with a silicon photodetector. In order to determine the reflectivity of the back mirrors, we measured both the threshold current and the differential quantum efficiency. Devices with microstructured back mirrors displayed a marked reduction in threshold current (Figs. 4 and 5) and an equally clear increase in differential quantum efficiency (Figs. 4 and 6). The fact that the shortest devices with etched facets did not lase at all [Fig. 4(a)], whereas the devices with microstructured mirrors had the lowest threshold for shortest cavities, is a further convincing demonstration of the quality of these microstructured mirrors.

\subsection{Threshold Current Density}

According to the standard model, the gain must equal the losses for a laser at threshold,

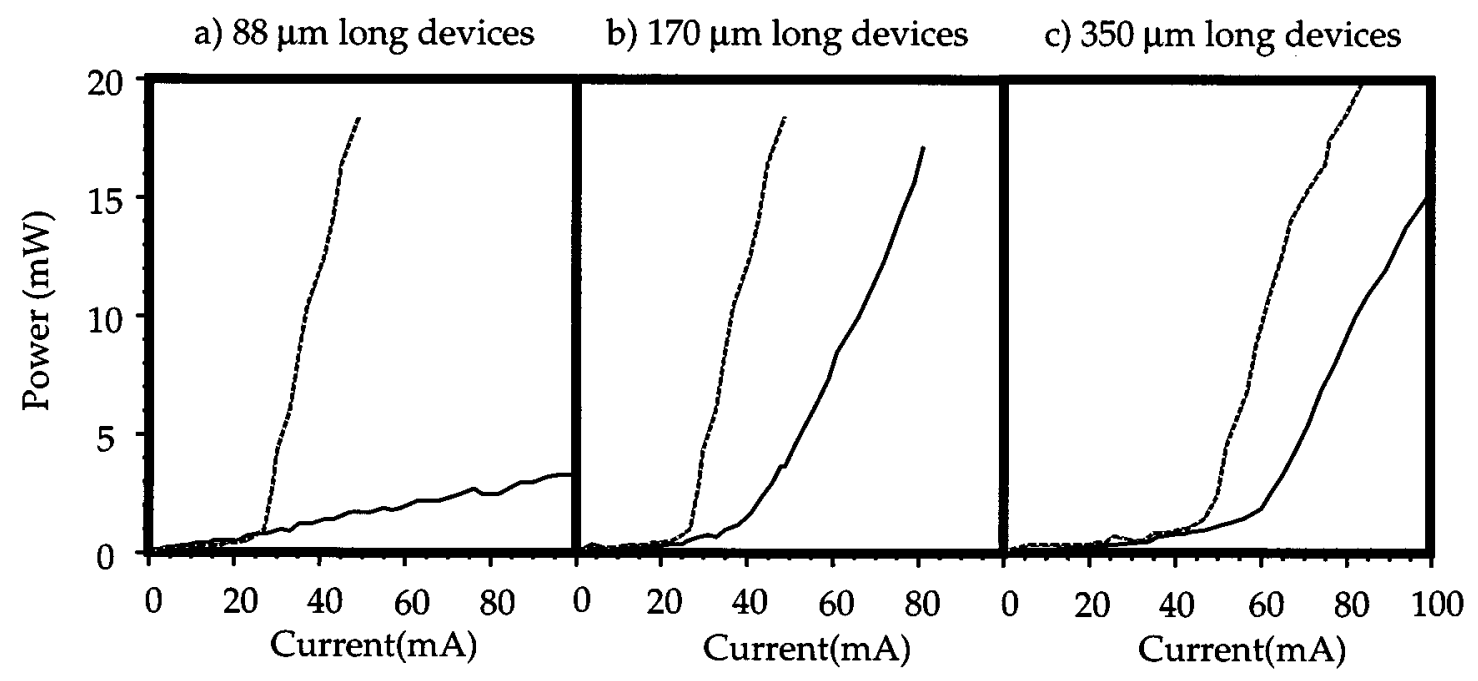

Fig. 4 L-I curves for three lasers with lengths (a) $88 \mu \mathrm{m}$, (b) $170 \mu \mathrm{m}$, and (c) $350 \mu \mathrm{m}$ for both dry-etched (solid line) and microstructured (broken line) back mirrors; the front mirror is a cleaved facet in all cases. The difference between the two types of back mirror is largest for the shortest devices (a), where the mirror loss is too high for the dry-etched device to achieve lasing threshold. The difference in threshold is smallest in (c), because the internal loss, which is the same in both types of device, begins to approach the mirror loss. 
Threshold current density vs. inverse length

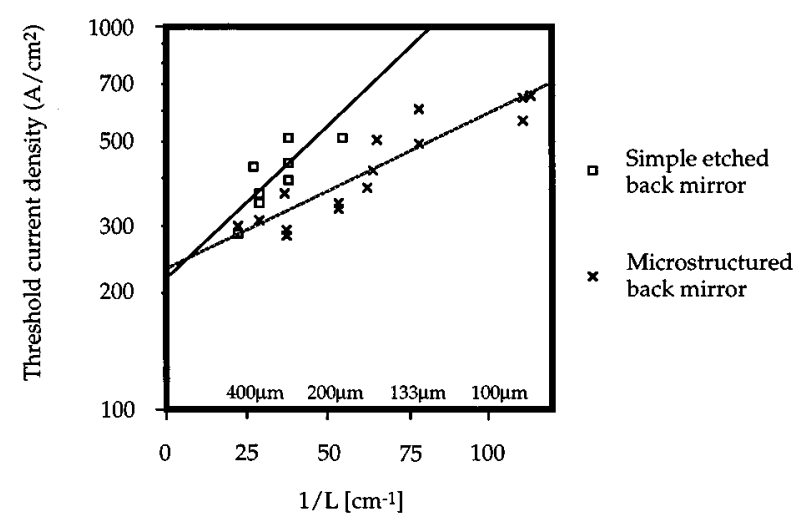

Fig. 5 Threshold current density versus inverse length for lasers with simple etched (solid line) and microstructured (broken line) mirrors.

$\Gamma g_{\mathrm{th}}=\alpha_{i}+\frac{1}{L} \ln \frac{1}{r_{1} r_{2}}$

where the threshold gain is denoted as $g_{\text {th }}$, the overlap factor between the optical mode and the quantum well is $\Gamma$, the internal losses are lumped together in $\alpha_{i}$, and the mirror loss $\alpha_{m}$ is $(1 / L) \ln \left(1 / r_{1} r_{2}\right)$. Here $L$ is the length of the resonator, and $r_{1}, r_{2}$ are the (amplitude) reflectivities of the two mirrors that form the cavity. In order to convert the gain of the quantum well to a threshold current, the logarithmic approximation ${ }^{11}$ is used, where the gain-current relationship is approximated by

$\frac{g}{g_{0}}=\ln \frac{J}{J_{0}}+1$

with $g$ representing the gain per well, $J$ the current density per well, and $g_{0}$ and $J_{0}$ parameters that depend on the well width. If Eqs. (1) and (2) are combined and suitably rear-

a) Inverse efficiency vs. length

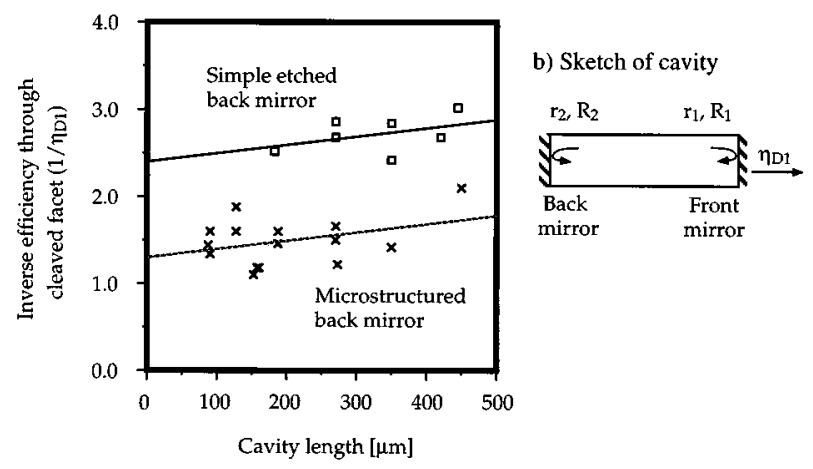

Fig. 6 (a) Inverse efficiency versus length for lasers with simple etched (solid line) and microstructured (broken line) back mirrors. The efficiency $\eta_{D 1}$ was measured through the front (i.e., cleaved) mirror, as indicated in the sketch (b). ranged, it can be shown that the slope $m_{J}$ in a graph of $\ln J_{\text {th }}$ versus $1 / L$ is directly proportional to the mirror reflectivity, or

$m_{J} \propto \ln \frac{1}{r_{1} r_{2}}$.

From Fig. 5, we obtain a ratio of 2.04 between the slopes for the two different types of mirror; assuming a value of $r=0.55(R=30 \%)$ for both the cleaved and the simple etched mirror and using Eq. (3), this yields $r_{2}$ $=1.0$, or $R_{2}=100 \%$, for the microstructured mirror.

\subsection{Differential Quantum Efficiency}

The reflectivity of the microstructured mirror can also be obtained via efficiency measurements. According to Ref. 12 , the differential quantum efficiency of a laser can be obtained from the output of a single facet as follows:

$\eta_{D 1}=F_{1} \eta_{i} \frac{\alpha_{m}}{\alpha_{m}+\alpha_{i}}$,

where $\eta_{D 1}$ represents the differential efficiency for light from mirror $1, F_{1}$ is the ratio of the power delivered out of mirror 1 to the total power coupled out of the cavity, $\eta_{i}$ is the internal quantum efficiency of the material, and $\alpha_{i}$ and $\alpha_{m}$ are the internal and mirror losses, respectively, as in Eq. (1). For lossless output mirrors, $F_{1}$ can be expressed as

$F_{1}=\frac{1-r_{1}^{2}}{1-r_{1}^{2}+\left(r_{1} / r_{2}\right)\left(1-r_{2}^{2}\right)}$.

In the special case of $r_{1}^{2}=r_{2}^{2}=R$ and $F_{1}=0.5$, Eq. (4) simplifies to the well-known relationship

$\frac{1}{\eta_{e x}}=\frac{1}{\eta_{i}}\left(\frac{\alpha_{i} L}{\ln (1 / R)}+1\right)$,

where $\eta_{e x}$ represents the total efficiency, i.e., the power coupled out of both mirrors. In order to obtain the individual mirror reflectivities, Eq. (4) can be rearranged as follows:

$$
\frac{1}{\eta_{D 1}}=\frac{1}{\eta_{i} F_{1}}+\frac{1}{\eta_{i} F_{1}} \frac{\alpha_{i}}{\ln \left(1 / r_{1} r_{2}\right)} L
$$

In a graph of $1 / \eta_{D 1}$ versus $L$, the slope yields the internal loss $\alpha_{i}$ if both mirror reflectivities are known; from the curve for the etched and cleaved devices, we obtain a value of $\alpha_{i}=9 \mathrm{~cm}^{-1}$. The slope does not yield any useful information on the microstructured and cleaved devices, however, because the product of the two factors that change with reflectivity, $F_{1}$ and $\ln \left(1 / r_{1} r_{2}\right)$, is virtually constant in the range $30 \%<R_{2}<100 \%$ (for $R_{1}$ fixed at $30 \%$ ). The information on the changing mirror reflectivity must then be obtained from the first term of Eq. (6), i.e., the intercept, which is directly proportional to $1 / F_{1}$. From Fig. 6(a), we 
get a ratio of 1.85 between the two intercepts, which, using Eq. (5), yields $r_{2}=0.95$, or $R=90 \%$ reflectivity, for the microstructured mirror.

In summary, both methods clearly show that the reflectivity of the microstructured mirror is significantly higher than that of a simple etched interface. We estimate the measurement error to be about $10 \%$, which gives a reflectivity of $(95 \pm 10) \%$ as the average of the two measurements.

\section{Discussion}

How realistic is the high reflectivity that we report, and why has it not been achieved before? Firstly, to put this value into perspective, let us compare it with the result obtained with a microcavity where a similar mirror structure to the one presented here was used. ${ }^{8}$ The value $Q$ $=2500$ that was measured there translates into a mirror reflectivity of $98 \%$, assuming a cavity length of $2 \mu \mathrm{m}$ and using the formula for the finesse of a Fabry-Perot cavity. ${ }^{13}$ This is a surprisingly similar result to the one reported here, so there is very reassuring agreement between passive and active measurements. Also, the regime of low transmission in the third-order stopband found in Ref. 8 corresponds to the high reflection found here, which emphasizes the fact that these high-contrast structures have low losses when designed properly. Secondly, the other reported work on such deeply etched Bragg mirrors ${ }^{3,4}$ uses "standard" laser material with a top cladding in excess of $1-\mu \mathrm{m}$ thickness, so the demands on the dry-etch process are considerably greater. Furthermore, and more importantly, Bragg mirrors with a high air fraction were used, so only a small part of the microstructure consists of waveguiding material. In this case, the light is more likely to scatter into the substrate and the air space above, i.e., the mirrors are probably more lossy than the ones presented here, which is why lower reflectivities were achieved.

Our data still hold even if the reflectivity of the simple dry-etched facet used for reference is, in reality, only $25 \%$ instead of the $30 \%$ we assumed in the analysis. The reflectivity of the microstructured facet then drops from $90 \%$ to $82 \%$ (efficiency data) and from $100 \%$ to $96 \%$ (threshold data), which is not a major deviation. As far as the waveguide design is concerned, the value of $9 \pm 1 \mathrm{~cm}^{-1}$ for the internal loss $\alpha_{i}$, derived from the slopes in Fig. 6(a) using Eq. (4), underlines the viability of the shallow-waveguide approach, even though we used simple $\mathrm{Ni}-\mathrm{Au}$ for the $p$-type (top) metallization instead of the more elaborate "shiny", contacts developed elsewhere. ${ }^{14,15}$

Further improvements are obvious: By using the narrower stripes of a normal single-mode laser, the threshold current can be decreased significantly and the fabricationrelated variations of the microstructured mirror across its width can be reduced, which should lead to a "cleaner", mirror response. By fabricating lasers with a microstructured front and back mirror, the thresholds well below 1 $\mathrm{mA}$ that have been predicted for devices of this type ${ }^{3}$ will come within reach. Additionally, photonic crystal mirrors can be added to the sides and VCSEL-type Bragg mirrors below the waveguide core, which will make it possible to curtail the photonic states of the cavity, i.e., reduce the number of modes into which the spontaneous emission can radiate. This will be the beginning of serious spontaneous- emission control and should lead to devices with even lower thresholds and very low-noise operation.

\section{Acknowledgments}

T.F.K. gratefully acknowledges support by the Royal Society of the UK. The work was funded through DARPA, the Army Research Office under contract DAAH04-96-1-0389, and the NSF under contract ECS-9632937.

\section{References}

1. G. M. Yang, M. H. MacDougal, and P. D. Dapkus, "Ultralow threshold current vertical-cavity surface-emitting lasers obtained with selective oxidation,' Electron. Lett. 31(11), 886-888 (1995).

2. E. Yablonovitch, "Inhibited spontaneous emission in solid-state physics and electronics," Phys. Rev. Lett. 58, 2059-2063 (1987).

3. T. Baba, M. Hamasaki, N. Watanabe, P. Kaewplungt, A. Matsutani, T. Mukaihara, F. Koyama, and K. Iga, "A novel short-cavity laser with deep-grating distributed Bragg reflectors,' Jpn. J. Appl. Phys. 1 35(2B), 1390-1394 (1996).

4. Y. Yuan, T. Brock, P. Bhattacharya, C. Caneau, and R. Bhat, "Edgeemitting lasers with short-period semiconductor/air distributed Bragg reflector mirrors,' IEEE Photonics Technol. Lett. 9(7), 881-883 (1997).

5. J. O'Brien, O. Painter, R. Lee, C. C. Cheng, A. Yariv, and A. Scherer, "Laser incorporating 2D photonic bandgap mirrors," Electron. Lett 32(24), 2243-2244 (1996).

6. D. Labilloy, H. Benisty, C. Weisbuch, T. F. Krauss, R. Houdre, and U. Oesterle, "Use of guided spontaneous emission of a semiconductor to probe the optical properties of two-dimensional photonic crystals,' Appl. Phys. Lett. 71(6), 738-740 (1997).

7. D. Labilloy, H. Benisty, C. Weisbuch, T. F. Krauss, R. M. De La Rue, V. Bardinal, R. Houdre, U. Oesterle, D. Cassagne, and C. Jouanin, "Quantitative measurement of transmission, reflection and diffraction of two-dimensional photonic bandgap structures at near-infrared wavelengths,'” PRL 79(21), 4147- 4150 (1997).

8. T. F. Krauss, B. Voegele, C. R. Stanley, and R. M. De La Rue, "Waveguide microcavity based on photonic microstructures," IEEE Photonics Technol. Lett. 9(2), 176-178 (1997).

9. T. F. Krauss, S. Brand, and R. M. De La Rue, "Two-dimensional photonic-bandgap structures operating at near-infrared wavelengths,' Nature 383, 699-702 (1996).

10. T. F. Krauss and R. M. De La Rue, "Optical characterisation of waveguide based photonic microstructures,"'Appl. Phys. Lett. 68(12), 1613-1615 (1996).

11. P. W. A. Mc Ilroy, A. Kurobe, and Y. Uematsu, "Analysis and Application of theoretical gain curves to the design of multi-quantumwell lasers,' IEEE J. Quantum Electron. QE-21(12), 1958-1963 (1985).

12. L. A. Coldren and S. W. Corzine, Diode Lasers and Photonic Integrated Circuits, p. 78, Wiley, New York (1995).

13. A. Yariv, Optical Electronics, Chap. 4, Saunders College Publ., Philadelphia (1993)

14. C. H. Wu, P. S. Zory, and M. A. Emanuel, "Characterisation of thin p-clad InGaAs single-quantum-well lasers,' IEEE Photonics Technol. Lett. 7(7), 718-720 (1995).

15. G. M. Smith, D. V. Forbes, R. M. Lammert, and J. J. Coleman, "Metallization to asymmetric cladding separate-confinement heterostructure lasers," Appl. Phys. Lett. 67(26), 3847-3849 (1995).

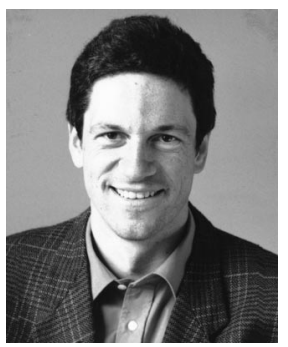

Thomas F. Krauss has been involved in research on laser diodes since 1989, when he took over research on ring resonator semiconductor lasers at Glasgow University under the direction of Professor P. J. R. Laybourn and revived interest worldwide in this topic through demonstrations of $\mathrm{cW}$ room temperature operation, low threshold current levels and greatly improved external device efficiency. In 1993, he obtained an EPSRC Research Fellowship for a proposal entitled "Photonic bandgap applied to semiconductor lasers," thereby initiating a new field at Glasgow University, followed by a five-year Royal Society Research Fellowship in the same area in 1995. Dr. Krauss was one of the first experimentalists in the United Kingdom to tackle the problems involved in making very small, high index contrast periodic structures in semiconductors, thereby showing the possibilities of the state-of-the-art technology available at Glasgow University. He recently achieved 
the first demonstration of a 2-D photonic bandgap (2-D PBG) in a semiconductor matrix at around $850-\mathrm{nm}$ wavelength as well as showing microcavity effects using a defect $(Q \sim 2500)$ in a 1-D PBG structure. He is currently on a one-year assignment at Caltech, Pasadena, California, working with Profs. Scherer and Yablonovitch on the realization of photonic crystal based light emitters.

Oskar Painter received the BEE degree from the University of British Columbia in 1994. Currently, he is working towards the PhD degree at the California Institute of Technology, Pasadena, California, where he is supported by a 1967 Science and Engineering Scholarship from the Natural Sciences and Engineering Research Council of Canada. His research is focused on the design and microfabrication of novel optical devices incorporating photonic bandgap crystals.

Axel Scherer is professor of electrical engineering, applied physics, and physics, specializing in device microfabrication and packaging. He graduated from New Mexico Institute of Mining and Technology in 1985, and worked in the Quantum Device Fabrication group at Bellcore for the following eight years. In the past, Dr. Scherer has specialized on improving the state of the art of semiconductor microfabrication, which resulted in the development of the smallest vertical cavity lasers (400 $\mathrm{nm}$ wide), some of the world's smallest etched structures ( $6 \mathrm{~nm}$ wide) as well as ultra-narrow gratings (30 $\mathrm{nm}$ pitch). He has also been working on reducing the sizes of microlasers and changing their emission wavelengths.

John S. Roberts received the BSc degree in applied chemistry in 1971 and the PhD degree on aspects of radiation chemistry in 1975. From 1975 to 1980, he was employed by Philips Research Laboratories, Redhill, United Kingdom, on developing MBE growth for III-V phosphides. Since 1980 he has been employed in the EPSRC Central Facility for III-V Semiconductors, University of Sheffield. He presently manages the MOVPE group with interests covering nitride-, phosphide-, and arsenide-based III-V materials.

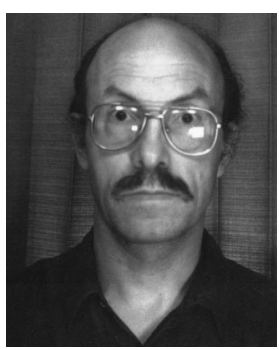

Richard M. De La Rue graduated with BScEng (Honors) in electrical engineering from University College, London (UCL), in 1966, subsequently obtaining the MASc degree in electrical engineering at the University of Toronto in 1968 and the $\mathrm{PhD}$ degree, also at UCL, in 1972. A paper based on his $\mathrm{PhD}$ work received the premium for the best paper published in Proceedings of IEE in 1972. In 1971, he was appointed as lecturer in the Department of Electronics and Electrical Engineering at the University of Glasgow, becom- ing senior lecturer in 1982, reader in 1985, and professor of optoelectronics in 1986. He spent a six-month period at Bell Labs, Murray Hill, New Jersey, in 1978, and three months as a Monbusho/ British Council sponsored lecturer at Tohoku University, Sendai, Japan, in 1980. He was elected fellow of the Royal Society of Edinburgh in 1989, is a member of IEE (UK) and OSA, and a senior member of IEEE. He is a member of the functional materials college of EPSRC. He is currently a visiting researcher at the Communications Research Laboratory, Tokyo, Japan. He has contributed significantly to a wide range of integrated optics research with a variety of applications. His recent work has been concerned in part with integration technology and devices based on III-V semiconductor quantum well heterostructures, including intermixing (QWI) processes to shift the refractive index and absorption edge, with DFB and DBR lasers using deep surface gratings and with mode-locked ring lasers. Professors De La Rue's research is now particularly concerned with photonic bandgap structures and microcavities. The importance of his work with Dr. T. F. Krauss on this topic is indicated by its publication in Nature. Current research also includes phase masks for photorefractive fiber gratings, periodic segmented waveguides in lithium niobate, and PBG effects in semiconductorloaded synthetic opal-a self-organized 3-D photonic crystal. He has published more than 200 articles and papers in journals, book chapters, and conference presentations. He was co-editor, with John H. Marsh, of Waveguide Optoelectronics, based on the 1990 NATO ASI held in Glasgow. 